PROCEEDINGS OF THE

AMERICAN MATHEMATICAL SOCIETY

Volume 133, Number 8, Pages 2347-2358

S 0002-9939(05)07920-7

Article electronically published on March 22, 2005

\title{
HYDRODYNAMIC APPROACH TO CONSTRUCTING SOLUTIONS OF THE NONLINEAR SCHRÖDINGER EQUATION IN THE CRITICAL CASE
}

\author{
O. S. ROZANOVA
}

(Communicated by Mark J. Ablowitz)

\begin{abstract}
Proceeding from the hydrodynamic approach, we construct exact solutions to the nonlinear Schrödinger equation with special properties. The solutions describe collapse, in finite time, and scattering, over infinite time, of wave packets. They generalize known blow-up solutions based on the "ground state".
\end{abstract}

\section{Preliminaries}

Consider the initial value problem for the nonlinear Schrödinger equation (NLS) in $\mathbb{R}^{n}$ :

$$
\begin{gathered}
i \Psi_{t}^{\prime}+\Delta \Psi+|\Psi|^{\sigma} \Psi=0, \quad \Psi(\mathbf{x}, t): \mathbb{R}_{x}^{n} \times \mathbb{R}_{t}^{+} \rightarrow \mathbb{C}, \\
\Psi(0, \mathbf{x})=\Psi_{0}(\mathbf{x}) \in H^{1}\left(\mathbb{R}^{n}\right) .
\end{gathered}
$$

It is well known that the Cauchy problem $(1),(2)$ has, locally in time, a solution of class $C\left([0, T) ; H^{1}\left(\mathbb{R}^{n}\right)\right), T \leq \infty[1],[2]$, [3]. The Cauchy problem is also locally well posed in $L^{2}\left(\mathbb{R}^{n}\right)[4$, and this space is optimal [5].

Moreover, $T=\infty$ for $\sigma<\frac{4}{n}$, where dispersion dominates, and for $\sigma \geq \frac{4}{n}$ the solution may "blow up" in finite time under certain initial conditions; e.g. [6], [7]. More exactly, there exist initial data $\Psi_{0}(\mathbf{x})$ and a positive constant $T\left(\Psi_{0}(\mathbf{x})\right)<\infty$, such that $\lim _{t \rightarrow T\left(\Psi_{0}(\mathbf{x})\right)} \int_{\mathbb{R}^{n}}|\nabla \Psi(\mathbf{x}, t)|^{2} d \mathbf{x}=\infty$.

The blow-up corresponds to self-trapping of beams in the laser propagation.

A very good review with references can be found in 8

For solutions to (1),(2) the following quantities are conserved:

$$
\begin{aligned}
& N[\Psi]=\|\Psi(\mathbf{x}, t)\|_{L_{2}\left(\mathbb{R}^{n}\right)}=\left\|\Psi_{0}(\mathbf{x})\right\|_{L^{2}\left(\mathbb{R}^{n}\right)}=N \quad \text { (the probability), } \\
& P[\Psi]=\operatorname{Im} \int_{\mathbb{R}^{n}} \Psi(\mathbf{x}, t) \nabla \bar{\Psi}(\mathbf{x}, t) d \mathbf{x}=P \quad \text { (the linear momentum), }
\end{aligned}
$$

and

$$
H[\Psi]=\|\nabla \Psi(\mathbf{x}, t)\|_{L^{2}\left(\mathbb{R}^{n}\right)}^{2}-\frac{2}{\sigma+2}\|\Psi(\mathbf{x}, t)\|_{L^{\sigma+2}\left(\mathbb{R}^{n}\right)}^{\sigma+2}=H \quad \text { (the energy). }
$$

Received by the editors December 10, 2003.

2000 Mathematics Subject Classification. Primary 35Q55; Secondary 35K55.

Key words and phrases. Nonlinear Schrödinger equation, hydrodynamic approach, integral functionals, exact solutions, blow-up solutions.

(C) 2005 American Mathematical Society Reverts to public domain 28 years from publication 2347 
Let $\sigma=\frac{4}{n}$. Assume additionally a stronger decay of initial data at infinity, namely, $|\mathbf{x}| \Psi_{0}(\mathbf{x}) \in L_{2}\left(\mathbb{R}^{n}\right)$. Then the following important identity holds:

$$
M^{\prime \prime}(t)=8 H,
$$

where $M(t)=\int_{\mathbb{R}^{n}}|\Psi(\mathbf{x}, t)|^{2}|\mathbf{x}|^{2} d \mathbf{x}$. This identity appears in many papers, but [9] seems to be the earliest one. It implies a very simple sufficient condition for the blow up. It is easy to see that $M(t)>0$ for the solution of class $C\left([0, T) ; H^{1}\left(\mathbb{R}^{n}\right)\right)$, $T \leq \infty$. At the same time (3) implies that

$$
M(t)=4 H t^{2}+M^{\prime}(0) t+M(0) .
$$

The blow up corresponds to vanishing of $M(t)$. It signifies the concentration of the solution support in a set of zero measure. From elementary algebra arguments we get that $M(t) \rightarrow 0$ at a finite moment of time $T$ if $K \leq 0$, where

$$
K=16 H M(0)-\left(M^{\prime}(0)\right)^{2} .
$$

This time $T$ is positive if $H<0$ or $H \geq 0, M^{\prime}(0)<0$.

In [10] it was proved that for radial initial conditions $\Psi_{0}(\mathbf{x})$ with $H<0$ the solution blows up in finite time in $H^{1}\left(\mathbb{R}^{n}\right)$ with no integrability conditions on $|\Psi|^{2}|\mathbf{x}|^{2}$ (see also [11] in the context).

\section{HydRodyNAMIC INTERPRETATION}

Below we use the hydrodynamic approach due to Madelung; e.g. [12. Namely, we represent the solution in trigonometric form, that is,

$$
\Psi(t, \mathbf{x})=A(t, \mathbf{x}) \exp (i \phi(t, \mathbf{x}))
$$

where $A(t, \mathbf{x}) \geq 0$ and $\phi(t, \mathbf{x})$ are real functions, the amplitude and the phase of the wave, respectively.

Note that if $A(t, \mathbf{x})$ is compactly supported, then $\Psi(t, \mathbf{x})$ is compactly supported, too. The support does not depend on the phase function $\phi$.

Substituting this representation in (1) and taking the real and imaginary parts of the resulting equation, we obtain the following system:

$$
\begin{aligned}
& A_{t}^{\prime}+2(\nabla A, \nabla \phi)+A \Delta \phi=0, \\
& A \phi_{t}^{\prime}+A|\nabla \phi|^{2}=\Delta A+A^{\sigma+1} .
\end{aligned}
$$

Further, we multiply (6) by $2 A$ and apply the gradient operator to (7). Denote by $\rho$ the probability density, $A^{2}=|\Psi|^{2}$, and by $V$ double the gradient of the phase function, $2 \nabla \phi$. Thus, the final hydrodynamic form of (1) consists of two equations

$$
\begin{gathered}
\rho_{t}^{\prime}+\rho \operatorname{div} V+(V, \nabla \rho)=0, \\
\rho\left(V_{t}^{\prime}+(V, \nabla) V\right)=2\left(A \nabla\left(\Delta A+A^{\sigma+1}\right)-\nabla A\left(\Delta A+A^{\sigma+1}\right)\right), \quad A=\rho^{1 / 2} .
\end{gathered}
$$

The only difference from the traditional gas dynamics is the "exotic" pressure on the right-hand side of (9). This type of pressure changes the character of singularity completely, but allows us to use the same methods as in the gas dynamics.

The data

$$
\rho(0, \mathbf{x})=\rho_{0}(\mathbf{x}), \quad V(0, \mathbf{x})=V_{0}(\mathbf{x})
$$

complete the statement of the Cauchy problem for (8),(9).

It suffices to demand that

$$
\rho_{0}^{1 / 2}(\mathbf{x}) \in H^{1}\left(\mathbb{R}^{n}\right), \quad \rho_{0}^{1 / 2}(\mathbf{x}) V_{0}(\mathbf{x}) \in L_{2}\left(\mathbb{R}^{n}\right),
$$


to ensure that the corresponding initial function $\Psi_{0}(\mathbf{x})$ belongs to the class $H^{1}\left(\mathbb{R}^{n}\right)$. Note also that $\rho$ vanishes as $|\mathbf{x}| \rightarrow \infty$, however, the same is not necessary for $V$. The conservation laws (I)-(III) in the new terms are as follows:

$$
\begin{gathered}
\int_{\mathbb{R}^{n}} \rho d \mathbf{x}=N \quad \text { (the mass), } \\
\int_{\mathbb{R}^{n}} \rho V d \mathbf{x}=\tilde{\mathbf{P}} \quad \text { (the linear momentum) }
\end{gathered}
$$

and

$$
\int_{\mathbb{R}^{n}}\left(\frac{\rho|V|^{2}}{4}+\frac{|\nabla \rho|^{2}}{4 \rho}-\frac{2}{\sigma+2} \rho^{\sigma / 2+1}\right) d \mathbf{x}=H \quad \text { (the energy). }
$$

One can see that the "kinetic energy" component in $H$ is the same as in gas dynamics (up to the multiplier).

Note that in gas dynamics terms we have

$$
M(t)=\int_{\mathbb{R}^{n}} \rho(\mathbf{x}, t)|\mathbf{x}|^{2} d \mathbf{x} .
$$

By virtue of (8),

$$
M^{\prime}(t)=2 \int_{\mathbb{R}^{n}}(\mathbf{x}, V(\mathbf{x}, t)) \rho(\mathbf{x}, t) d \mathbf{x}
$$

here $\mathbf{x}$ is the radius-vector of a point in the space.

Let us introduce one more functional:

$$
Q_{\Lambda}(t)=\int_{\mathbb{R}^{n}}(\mathbf{x}, \boldsymbol{\Lambda}) \rho(\mathbf{x}, t) d \mathbf{x},
$$

where $\boldsymbol{\Lambda}$ is a constant vector from $\mathbb{R}^{n}$. From (8) and the conservation of linear momentum $\left(\mathrm{II}^{\prime}\right)$ we have $Q_{\Lambda}^{\prime}(t)=(\tilde{\mathbf{P}}, \boldsymbol{\Lambda}):=\tilde{\mathbf{P}}_{\boldsymbol{\Lambda}}=$ const, and

$$
Q_{\Lambda}(t)=\tilde{\mathbf{P}}_{\Lambda} t+Q_{\Lambda}(0) .
$$

From the Hölder inequality we also have

$$
Q_{\boldsymbol{\Lambda}}^{2}(t) \leq|\boldsymbol{\Lambda}|^{2} N M(t) .
$$

In the domains where the amplitude $A>0$, instead of (9) we consider the equivalent equation

$$
V_{t}^{\prime}+(V, \nabla) V=2 \nabla\left(\frac{\Delta A}{A}+A^{\sigma}\right) .
$$

Now we use the following idea, recently applied to construct solutions to the gas dynamics equations (see [13], [14], [15], [16]). Namely, let the "velocity field" have the form

$$
V=a(t) \mathbf{x},
$$

where $a(t)$ is a time-dependent function. Thus, the phase function can be restored as

$$
\phi(t, \mathbf{x})=a(t) \frac{|\mathbf{x}|^{2}}{4}+\gamma(t),
$$


with an unknown function $\gamma(t)$. Then, from the (linear in $\rho$ ) equation (8) we find the density as

$$
\rho(t, \mathbf{x})=\exp \left(-n \int_{0}^{t} a(\tau) d \tau\right) \rho_{0}\left(\mathbf{x} \exp \left(-\int_{0}^{t} a(\tau) d \tau\right)\right)
$$

or

$$
A(t, \mathbf{x})=\exp \left(-\frac{n}{2} \int_{0}^{t} a(\tau) d \tau\right) A_{0}\left(\mathbf{x} \exp \left(-\int_{0}^{t} a(\tau) d \tau\right)\right)
$$

with $A_{0}(\mathbf{x})=\left|\Psi_{0}(\mathbf{x})\right|$.

Note that in the critical case, $\sigma=\frac{4}{n}$,

$$
\frac{\Delta A(t, \mathbf{x})}{A(t, \mathbf{x})}+A^{\sigma}(t, \mathbf{x})=\exp \left(-2 \int_{0}^{t} a(\tau) d \tau\right)\left(\frac{\Delta A_{0}(\xi)}{A_{0}(\xi)}+A_{0}^{\sigma}(\xi)\right)
$$

where $\xi=\mathbf{x} \exp \left(-\int_{0}^{t} a(\tau) d \tau\right)$.

Further, we have from (7) and (12)

$$
\left(a^{\prime}(t)+a^{2}(t)\right) \frac{|\mathbf{x}|^{2}}{4}+\gamma^{\prime}(t)=\exp \left(-2 \int_{0}^{t} a(\tau) d \tau\right)\left(\frac{\Delta A_{0}(\xi)}{A_{0}(\xi)}+A_{0}^{\sigma}(\xi)\right)
$$

or

$\left(a^{\prime}(t)+a^{2}(t)\right) \frac{|\xi|^{2}}{4}+\gamma^{\prime}(t) \exp \left(-2 \int_{0}^{t} a(\tau) d \tau\right)=\exp \left(-4 \int_{0}^{t} a(\tau) d \tau\right)\left(\frac{\Delta A_{0}(\xi)}{A_{0}(\xi)}+A_{0}^{\sigma}(\xi)\right)$.

The variables $t$ and $\xi$ can be separated if

$$
\gamma^{\prime}(t)=\gamma_{0} \exp \left(-2 \int_{0}^{t} a(\tau) d \tau\right), \quad \gamma_{0} \in \mathbb{R}^{1} .
$$

In this case

$$
\left(a^{\prime}(t)+a^{2}(t)\right)|\xi|^{2}=4 \exp \left(-4 \int_{0}^{t} a(\tau) d \tau\right)\left(\frac{\Delta A_{0}(\xi)}{A_{0}(\xi)}+A_{0}^{\sigma}(\xi)-\gamma_{0}\right) .
$$

It follows from (14) that

$$
\begin{gathered}
a^{\prime}(t)+a^{2}(t)=4 k \exp \left(-4 \int_{0}^{t} a(\tau) d \tau\right) \\
\Delta A_{0}(\mathbf{x})+A_{0}^{\sigma+1}(\mathbf{x})=\left(k|\mathbf{x}|^{2}+\gamma_{0}\right) A_{0}(\mathbf{x})
\end{gathered}
$$

where $k$ is a constant. 
Thus, we seek a special solution to (1) in the form

$$
\begin{gathered}
\Psi(t, \mathbf{x})=\exp \left(-\frac{n}{2} \int_{0}^{t} a(\tau) d \tau\right) A_{0}\left[\mathbf{x} \exp \left(-\int_{0}^{t} a(\tau) d \tau\right)\right] \\
\times \exp \left(i a(t) \frac{|\mathbf{x}|^{2}}{4}\right) \exp \left(i \gamma_{0} \int_{0}^{t} \exp \left(-2 \int_{0}^{\tau} a\left(\tau_{1}\right) d \tau_{1}\right) d \tau\right) \exp (i \theta),
\end{gathered}
$$

with $\theta \in \mathbb{R}^{1}$.

Because the origin is not a particular point, without loss of generality we may consider the velocity field $V=a(t)\left(\mathbf{x}-\mathbf{x}_{0}\right)$, where $\mathbf{x}_{0}$ is an arbitrary fixed point. Then the solution takes the form

$$
\begin{gathered}
\Psi(t, \mathbf{x})=\exp \left(-\frac{n}{2} \int_{0}^{t} a(\tau) d \tau\right) A_{0}\left[\left(\mathbf{x}-\mathbf{x}_{0}\right) \exp \left(-\int_{0}^{t} a(\tau) d \tau\right)\right] \\
\times \exp \left(i a(t) \frac{\left|\mathbf{x}-\mathbf{x}_{0}\right|^{2}}{4}\right) \exp \left(i \gamma_{0} \int_{0}^{t} \exp \left(-2 \int_{0}^{\tau} a\left(\tau_{1}\right) d \tau_{1}\right) d \tau\right) \exp (i \theta) .
\end{gathered}
$$

The decay properties of the solution as $|\mathbf{x}| \rightarrow \infty$ depend on $A_{0}(\mathbf{x})$. The physical sense requires that the solution should be of the class $L^{2}\left(\mathbb{R}^{n}\right)$. If we wish to consider solutions from the space $C\left([0, T) ; H^{1}\left(\mathbb{R}^{n}\right)\right), T \leq \infty$, natural for the existence and uniqueness to the Cauchy problem (1), (2), we have to choose the initial data

$$
\Psi_{0}(\mathbf{x})=A_{0}\left(\mathbf{x}-\mathbf{x}_{0}\right) \exp \left(i a_{0} \frac{\left|\mathbf{x}-\mathbf{x}_{0}\right|^{2}}{4}\right) \exp (i \theta)
$$

where $A_{0}(\mathbf{x})$ is a nonnegative solution to (14) belonging to $H^{1}\left(\mathbb{R}^{n}\right)$ and $|\mathbf{x}| A_{0}(\mathbf{x}) \in$ $L_{2}\left(\mathbb{R}^{n}\right)$. Note that with the function $A_{0}$ of this class we also have conservation laws (I)-(III) for solutions (17) and $\left(17^{\prime}\right)$.

\section{Time Evolution}

Let us investigate the qualitative behavior of $a(t)$ governed by (15). It is easy to see that in the case $k<0$ for any initial datum $a(0)=a_{0}$ there exists a moment $T_{*}$ such that $a(t) \rightarrow-\infty, t \rightarrow T_{*}$. Really, as $a^{\prime}(t)<\epsilon<0$, then under any $a_{0}$ there exists a moment $T_{1} \geq 0$ when $a\left(T_{1}\right)<0$. Further, the comparison theorem shows that $a(t) \leq \tilde{a}(t)$, where $\tilde{a}(t)$ is a solution to the Cauchy problem $\tilde{a}(t)^{\prime}=$ $-\tilde{a}^{2}(t), \tilde{a}\left(T_{1}\right)=a\left(T_{1}\right), t \geq T_{1}$. This means that if $k<0$, the solution $\Psi$ to (1) of the form (17), with the amplitude $A_{0}$ satisfying (16), localizes at the origin within a finite interval of time, provided $N<\infty$.

We can also analyze the differential corollary of (15)

$$
a^{\prime \prime}(t)+6 a(t) a^{\prime}(t)+4 a^{3}(t)=0,
$$

with the initial data $a(0)=a_{0}, a^{\prime}(0)=-a_{0}^{2}+4 k$. This gives, in particular, that in the case $k>0$ the solution vanishes at infinity as $O\left(t^{-1}\right)$.

If $k=0$, any nontrivial solution to (15) blows up at a finite time $T=-a_{0}^{-1}$. The time is positive if $a_{0}<0$. Moreover, if $|\mathbf{x}| \Psi_{0}(\mathbf{x}) \in L^{2}\left(\mathbb{R}^{n}\right)$, we can find $a(t)$ explicitly for any $k$. Observe that, if $V=a(t) \mathbf{x}$, then

$$
M^{\prime}(t)=2 a(t) M(t),
$$


therefore $a(t)=\frac{M^{\prime}(t)}{2 M(t)}$. The explicit form of $M(t)$ is known; see (4). Thus,

$$
a(t)=\frac{8 H t+M^{\prime}(0)}{2\left(4 H t^{2}+M^{\prime}(0) t+M(0)\right)} .
$$

If $K=16 H M(0)-\left(M^{\prime}(0)\right)^{2} \leq 0$ (see (5)), then $M(t)$ tends to zero $(a(t)$ goes to infinity, respectively) within a finite interval of time. Moreover, this interval can be readily calculated. If $K>0$, then $a(t) \sim t^{-1}, t \rightarrow \infty$.

Now taking into account (15), (18), (19) we can compute

$$
k=\frac{K}{16 M^{2}(0)}=\frac{16 H M(0)-\left(M^{\prime}(0)\right)^{2}}{16 M^{2}(0)} .
$$

There are situations where we can express $a(t)$ through $Q_{\boldsymbol{\Lambda}}(t)$. Namely, for $V=$ $a(t) \mathbf{X}$ we get $\tilde{\mathbf{P}}_{\boldsymbol{\Lambda}}=a(t) Q_{\boldsymbol{\Lambda}}(t)$. Therefore, if there is $\boldsymbol{\Lambda} \in \mathbb{R}^{n}$ such that $Q_{\boldsymbol{\Lambda}}(t) \neq$ $0\left(\tilde{\mathbf{P}}_{\boldsymbol{\Lambda}} \neq 0\right)$, then taking into account $(10)$ we have

$$
a(t)=\frac{1}{t+Q_{\Lambda}(0) \tilde{\mathbf{P}}_{\boldsymbol{\Lambda}}^{-1}}=\frac{1}{t+a_{0}^{-1}} .
$$

From (18) we now obtain $M(t)=M(0) a_{0}^{2}\left(t+a_{0}^{-1}\right)^{2}$. Comparing the result with (4) we can see that $4 H=M(0) a_{0}^{2}$ and $K=k=0$.

Note that if $A_{0}$ is radial, then $Q_{\boldsymbol{\Lambda}}(t)=0$ for any $\boldsymbol{\Lambda}$.

\section{Evolution of WAVE PACKets}

Summarizing the above results, we can formulate the following theorem:

Theorem 1. Suppose that (16) has at least one nonnegative solution $A_{0}(\mathbf{x})$. Then equation (1) has a special solution given by the explicit formula $(17)\left(\left(17^{\prime}\right)\right)$, with the function a $(t)$ governed by equation (15).

If $\mathbf{x} A_{0}(\mathbf{x}) \in L^{2}\left(\mathbb{R}^{n}\right)$, then the formula $\left(17^{\prime}\right)$ can be written as

$$
\begin{gathered}
\Psi(t, \mathbf{x})=\left(\frac{M(0)}{M(t)}\right)^{\frac{n}{4}} A_{0}\left[\left(\frac{M(0)}{M(t)}\right)^{\frac{1}{2}}\left(\mathbf{x}-\mathbf{x}_{0}\right)\right] \\
\times \exp \left(i \frac{M^{\prime}(t)}{8 M(t)}\left|\mathbf{x}-\mathbf{x}_{0}\right|^{2}\right) \exp \left(i \gamma_{0} M(0) \int_{0}^{t} M^{-1}(\tau) d \tau\right) \exp (i \theta),
\end{gathered}
$$

with the quadratic function $M(t)$ having the explicit form (4).

The behavior of the solution depends on the sign of the constant $k$ (see (20)).

If $k>0$, then the solution decays. Namely,

$$
\max _{\mathbf{x} \in \mathbb{R}^{n}}|\Psi(t, \mathbf{x})|=A^{+}\left(\frac{M(0)}{4 H}\right)^{\frac{n}{4}} t^{-n / 2}, \quad A^{+}=\max _{\mathbb{R}^{n}} A_{0}(\mathbf{x}) .
$$

If $k \leq 0$, then the solution blows up at the point $\mathbf{x}_{0}$ at a finite moment of time $T$. This time is positive in the following cases:

(i) if $H=0, M^{\prime}(0)<0$, then $T=\frac{-M(0)}{M^{\prime}(0)}$;

(ii) if $H>0, M^{\prime}(0)<0$, then $T=\frac{-M^{\prime}(0)-\sqrt{|K|}}{2 H}$;

(iii) if $H<0$, then $T=\frac{-M^{\prime}(0)+\sqrt{|K|}}{2 H}$.

Moreover, for $k=0$,

$$
\max _{\mathbf{x} \in \mathbb{R}^{n}}|\Psi(t, \mathbf{x})|=A^{+}\left(\frac{M(0)}{4 H}\right)^{\frac{n}{4}}(T-t)^{-n / 2},
$$


and for $k<0$,

$$
\max _{\mathbf{x} \in \mathbb{R}^{n}}|\Psi(t, \mathbf{x})|=A^{+}\left(\frac{M(0)}{\sqrt{|K|}}\right)^{\frac{n}{4}}(T-t)^{-n / 4} .
$$

Remark 1. If there exists $\boldsymbol{\Lambda} \in \mathbb{R}^{n}$ such that $Q_{\boldsymbol{\Lambda}}(t) \neq 0\left(\tilde{\mathbf{P}}_{\boldsymbol{\Lambda}} \neq 0\right)$, then only the situation with $k=0$ may be realized.

Remark 2. For the solution of the form (19) we have

$$
\begin{gathered}
\|\nabla \Psi(\mathbf{x}, t)\|_{L^{2}\left(\mathbb{R}^{n}\right)}^{2}=\exp \left(-2 \int_{0}^{t} a(\tau) d \tau\right)\left\|\nabla A_{0}(\mathbf{x})\right\|_{L^{2}\left(\mathbb{R}^{n}\right)}^{2} \\
+\frac{1}{4} a^{2}(t) \exp \left(2 \int_{0}^{t} a(\tau) d \tau\right)\left\|\mathbf{x} A_{0}(\mathbf{x})\right\|_{L^{2}\left(\mathbb{R}^{n}\right)}^{2} \\
=\frac{M(0)}{M(t)}\left\|\nabla A_{0}(\mathbf{x})\right\|_{L_{2}\left(\mathbb{R}^{n}\right)}^{2}+\frac{\left(M^{\prime}(t)\right)^{2}}{16 M(t)}=O(T-t)^{-\lambda}
\end{gathered}
$$

where $\lambda=1$ (the lower estimate for the blow-up order; see [17]) for $k<0$, and $\lambda=2$ for $k=0$.

\section{COMPARISON With PREvious RESUlts}

In the theory of NLS for the critical case $\sigma=\frac{4}{n}$ the crucial role is played by the so-called ground state, i.e. the positive radially-decreasing solution to the elliptic problem

$$
\Delta u+|u|^{\sigma} u-u=0, u \in H^{1}\left(\mathbb{R}^{n}\right) .
$$

It is known [18 that the solution with such properties is unique and exists at least for $n=1,2,3$. The solution belongs to $C^{2}\left(\mathbb{R}^{n}\right)$, and $\left|D^{\alpha} u\right| \leq C \exp (-\delta|x|)$, where $C, \delta$ are positive constants. We denote it by $R(\mathbf{x})$.

It is known that $H[R(\mathbf{x})]=0[19$.

It was proved [19] that if $\left\|\Psi_{0}(\mathbf{x})\right\|_{L^{2}\left(\mathbb{R}^{n}\right)}<\|R(\mathbf{x})\|_{L^{2}\left(\mathbb{R}^{n}\right)}$, then the solution to the problem (1), (2) is global in time. If the solution blows up, then $\left\|\Psi_{0}(\mathbf{x})\right\|_{L_{2}\left(\mathbb{R}^{n}\right)}>$ $\|R(\mathbf{x})\|_{L^{2}\left(\mathbb{R}^{n}\right)}$. In the case $\left\|\Psi_{0}(\mathbf{x})\right\|_{L^{2}\left(\mathbb{R}^{n}\right)}=\|R(\mathbf{x})\|_{L^{2}\left(\mathbb{R}^{n}\right)}$ the solution either blows up or not.

If it does blow up, it necessarily has the following special form based on the ground state [20] 21]:

$$
\Psi(\mathbf{x}, t)=\exp (i \theta) \exp \left(i\left(-\frac{\omega^{2}}{t-T}+\frac{\left|\mathbf{x}-\mathbf{x}_{0}\right|^{2}}{4(t-T)}\right)\right)\left(\frac{\omega}{t-T}\right)^{\frac{n}{2}} R\left(\frac{\omega\left(\mathbf{x}-\mathbf{x}_{0}\right)}{t-T}-\mathbf{x}_{1}\right),
$$

with certain $\mathbf{x}_{0} \in \mathbb{R}^{n}, \mathbf{x}_{1} \in \mathbb{R}^{n}, \theta \in \mathbb{R}, \omega \in \mathbb{R}_{+}, T \in \mathbb{R}$. However, (23) coincides with (21) for $K=k=0$ and $\gamma_{0}=1$. Indeed, in this case $R(x)$ is a solution to (14), $M(t)=H\left(t+\frac{M^{\prime}(0)}{2 H}\right)^{2}=H\left(t-\left(-\frac{M(0)}{H}\right)^{\frac{1}{2}}\right)^{2}, \omega^{2}=\frac{M(0)}{H}, T=\omega=-\frac{M^{\prime}(0)}{2 H}$. Here we choose $M^{\prime}(0)<0$ to guarantee the positivity of $T$. Note that $H>0$ for solutions of the form (23). 
It can be readily demonstrated that

$$
\begin{gathered}
H[\Psi]=\frac{M(0)}{M(t)}\left(\left\|\nabla A_{0}(\mathbf{x})\right\|_{L_{2}\left(\mathbb{R}^{n}\right)}^{2}-\frac{2}{\sigma+2}\left\|\nabla A_{0}(\mathbf{x})\right\|_{L_{\sigma+2}\left(\mathbb{R}^{n}\right)}^{\sigma+2}\right)+\frac{\left(M^{\prime}(t)\right)^{2}}{16 M(t)} \\
=\frac{M(0)}{M(t)} H\left[A_{0}\right]+\frac{\left(M^{\prime}(t)\right)^{2}}{16 M(t)} .
\end{gathered}
$$

Thus, for $A_{0}=R$, we get $H=\frac{\left(M^{\prime}(t)\right)^{2}}{16 M(t)}>0$.

It follows from the above results that if $A_{0}(\mathbf{x})$ is a solution to (16) with $k<0$ from $H^{1}\left(\mathbb{R}^{n}\right)$, then

$$
\left\|A_{0}(\mathbf{x})\right\|_{L^{2}\left(\mathbb{R}^{n}\right)}>\|R(\mathbf{x})\|_{L^{2}\left(\mathbb{R}^{n}\right)},
$$

because in this case the solution (17) blows up and it is not of the form (23).

The profile of our solution in the case $k<0$ is different from that of the solution (23) (corresponding to $k=0$ ), and so is the rate of blow up (see Remark 2 of Section 4).

Note that in the case $k<0$ the solution to equation (16), considered in the space $\mathbb{R}^{n}$, oscillates as $|x| \rightarrow \infty$. For the linearized equation $(n=1)$ we can even get the explicit solution:

$$
u=|x|^{-1 / 2}\left(C_{1} W_{1}\left(\frac{i \gamma_{0}}{2 \sqrt{2 k}}, \frac{1}{4}, \frac{1}{2} i \sqrt{2 k} x^{2}\right)+C_{2} W_{2}\left(\frac{i \gamma_{0}}{2 \sqrt{2 k}}, \frac{1}{4}, \frac{1}{2} i \sqrt{2 k} x^{2}\right)\right),
$$

where $W_{1}, W_{2}$ are the Whittaker functions, $C_{1}, C_{2}$ are constants. This highly oscillating function does not belong to $L^{2}\left(\mathbb{R}^{n}\right)$. So we cannot hope that the solution to nonlinear perturbed problem (16) is positive and belongs to $L^{2}\left(\mathbb{R}^{n}\right)\left(H^{1}\left(\mathbb{R}^{n}\right)\right)$.

However, we can consider (in higher dimensions, too) the solution to (16) given in $\Omega \subset \mathbb{R}^{n}$. To be exact, now we deal with the Dirichlet problem for (16) with zero boundary conditions. Regarding this Dirichlet problem, for example, there is the following result due to 22 .

Theorem 2 (22]). Let $\Omega$ be a bounded, smooth domain in $\mathbb{R}^{n}, n \geq 2$, and let $g: \mathbb{R}^{+} \times \bar{\Omega} \rightarrow \mathbb{R}^{+}$be a locally Lipshitzian map. Consider the elliptical boundary problem

$$
\Delta u+u^{p}+\epsilon g(x, u)=0,\left.u\right|_{\partial \Omega}=0 .
$$

If $1<p<\frac{n+1}{n-2}(p>1$ for $n=1,2)$, there exists $\epsilon_{0}>0$ such that, for $0 \leq \epsilon<\epsilon_{0}$, (*) has a solution $u=u_{\epsilon}$, which is positive on $\Omega$.

In our situation $g(x, u)=\frac{-k|x|^{2}-\gamma_{0}}{\epsilon} u$ satisfies the theorem condition for $k<0$ in a ball from $\mathbb{R}^{n}$; for $\gamma_{0}<0$ this condition holds for all $\mathbb{R}^{n}$.

The solution to the Dirichlet problem is classical, that is, it belongs to $C^{2}(\Omega) \cap$ $C(\bar{\Omega})$, therefore it can be extended to the whole space $\mathbb{R}^{n}$, at least as a solution from $L^{2}\left(\mathbb{R}^{n}\right)$.

\section{FURTHER GenERALIZATION}

Let us consider the following velocity field:

$$
\mathbf{V}=a(t) \mathbf{x}+b(t) \mathbf{\Lambda},
$$

with a constant vector $\boldsymbol{\Lambda}$. Then the phase function takes the form

$$
\phi(t, \mathbf{x})=a(t) \frac{|\mathbf{x}|^{2}}{4}+\frac{1}{2} b(t)(\Lambda, x)+\gamma(t) .
$$


Proceeding in the spirit of Section 2, we obtain

$$
\begin{aligned}
A(t, \mathbf{x})=\exp \left(-\frac{n}{2} \int_{0}^{t} a(\tau) d \tau\right) A_{0}(\mathbf{x} \exp ( & \left.-\int_{0}^{t} a(\tau) d \tau\right) \\
& \left.-\boldsymbol{\Lambda} \int_{0}^{t} b(\tau) \exp \left(-\int_{0}^{\tau} a\left(\tau_{1}\right) d \tau_{1}\right) d \tau\right) .
\end{aligned}
$$

Denote $\xi=\mathbf{x} \exp \left(-\int_{0}^{t} a(\tau) d \tau\right)-\boldsymbol{\Lambda} \int_{0}^{t} b(\tau) \exp \left(-\int_{0}^{\tau} a\left(\tau_{1}\right) d \tau_{1}\right) d \tau$. From (7) and (12) we get

$$
\begin{gathered}
{\left[a^{\prime}(t)+a^{2}(t)\right] \frac{|\xi|^{2}}{4}} \\
+\left[\left(a^{\prime}(t)+a^{2}(t)\right) \int_{0}^{t} b(\tau) \exp \left(-\int_{0}^{\tau} a\left(\tau_{1}\right) d \tau_{1}\right) d \tau\right. \\
\left.+\left(b^{\prime}(t)+a(t) b(t)\right) \exp \left(-\int_{0}^{t} a(\tau) d \tau\right)\right] \frac{(\boldsymbol{\Lambda}, \mathbf{x})}{2} \\
+\left[\left(a^{\prime}(t)+a^{2}(t)\right)\left(\int_{0}^{t} b(\tau) \exp \left(-\int_{0}^{\tau} a\left(\tau_{1}\right) d \tau_{1}\right) d \tau\right)^{2}\right. \\
+2\left(b^{\prime}(t)+a(t) b(t)\right) \exp \left(-\int_{0}^{t} a(\tau) d \tau\right) \int_{0}^{t} b(\tau) \exp \left(-\int_{0}^{\tau} a\left(\tau_{1}\right) d \tau_{1}\right) d \tau \\
\left.+b^{2}(t) \exp \left(-2 \int_{0}^{t} a(\tau) d \tau\right)\right] \frac{|\boldsymbol{\Lambda}|^{2}}{4} \\
-\gamma^{\prime}(t) \exp \left(-2 \int_{0}^{t} a(\tau) d \tau\right)=\exp \left(-4 \int_{0}^{t} a(\tau) d \tau\right)\left(\frac{\Delta A_{0}(\xi)}{A_{0}(\xi)}+A_{0}^{\sigma}(\xi)\right) .
\end{gathered}
$$

There are two possibilities for the separation of variables.

I. The functions $a(t), \gamma(t)$ and $A_{0}\left(\mathbf{x}_{1}\right), \mathbf{x}_{1}=\mathbf{x}+\boldsymbol{\Lambda}$ satisfy equations (15), (13) and (16), respectively, $b(t)=a(t)$. So we return to the formula (17') considered above.

II. The functions $a(t), b(t), \gamma(t)$ and $A_{0}(\mathbf{x})$ satisfy the following equations:

$$
\begin{gathered}
a^{\prime}(t)+a^{2}(t)=0 \\
b^{\prime}(t)+a(t) b(t)=2 k_{1} \exp \left(-3 \int_{0}^{t} a(\tau) d \tau\right) \\
\gamma^{\prime}(t)=\gamma_{0} \exp \left(-2 \int_{0}^{t} a(\tau) d \tau\right) \\
-\left[4 k_{1} \exp \left(-2 \int_{0}^{t} a(\tau) d \tau\right)+\int_{0}^{t} b(\tau) \exp \left(-\int_{0}^{\tau} a\left(\tau_{1}\right) d \tau_{1}\right) d \tau+b^{2}(t)\right] \frac{|\boldsymbol{\Lambda}|^{2}}{4} \\
\Delta A_{0}(\mathbf{x})+A_{0}^{\sigma+1}(\mathbf{x})=\left(k_{1}(\boldsymbol{\Lambda}, \mathbf{x})+\gamma_{0}\right) A_{0}(\mathbf{x}),
\end{gathered}
$$

where $\gamma_{0}$ and $k_{1}$ are constants.

The functions $a(t), b(t), \gamma(t)$ can be found explicitly.

Note that if $k_{1}=0, \gamma_{0}=1, b(t)=0$, we again obtain a solution that can be represented by formula $(23)$. 
The simplest situation is $a(t)=0, b(t)=2 k_{1} t+b_{0}, \gamma(t)=-\frac{|\boldsymbol{\Lambda}|^{2}}{12 k_{1}}\left(2 k_{1} t+b_{0}\right)^{3}+$ $\gamma_{0} t+\gamma_{1}, \gamma_{0}, \gamma_{1} \in \mathbb{R}^{n}$. The corresponding solution has the form

$$
\Psi(t, \mathbf{x})=A_{0}\left[\mathbf{x}-\mathbf{\Lambda} t\left(k_{1} t+b_{0}\right)\right] \exp \left(i\left(k_{1} t+\frac{b_{0}}{2}\right)(\boldsymbol{\Lambda}, \mathbf{x})+\gamma(t)\right),
$$

with the function $A_{0}(\mathbf{x})$ satisfying (28). For $k_{1}=0, \gamma_{0}=1, b_{0}=0$ we get the solitary wave solution $R(\mathbf{x}) \exp \left(i\left(t+\gamma_{1}\right)\right)$.

In the general case

$$
\begin{aligned}
a(t) & =\frac{1}{t+a_{0}^{-1}}, \\
b(t) & =\frac{b_{0} a_{0}^{-1}+2 k_{1} a_{0}^{2}}{t+a_{0}^{-1}}-2 k_{1} \frac{1}{\left(t+a_{0}^{-1}\right)^{2}}, \\
\gamma(t) & =\int_{0}^{t}\left[\frac{\gamma_{0}}{\left(\tau+a_{0}^{-1}\right)^{-2}}+\frac{k_{1}|\boldsymbol{\Lambda}|^{2}}{\tau+a_{0}^{-1}} \int_{0}^{\tau} \frac{b\left(\tau_{1}\right)}{\tau_{1}+a_{0}^{-1}} d \tau_{1}-\frac{1}{4}|\boldsymbol{\Lambda}|^{2} b^{2}(\tau)\right] d \tau .
\end{aligned}
$$

The corresponding solution has the form

$$
\begin{gathered}
\Psi(\mathbf{x}, t)=\exp (i \gamma(t)) \exp \left(i\left(b(t) \frac{(\mathbf{x}, \boldsymbol{\Lambda})}{2}+\frac{|\mathbf{x}|^{2}}{4\left(t+a_{0}^{-1}\right)}\right)\right) \\
\left(\frac{1}{\left|t+a_{0}^{-1}\right|}\right)^{\frac{n}{2}} A_{0}\left(\frac{\mathbf{x}}{t+a_{0}^{-1}}-\boldsymbol{\Lambda} \int_{0}^{t} \frac{b(\tau)}{t+a_{0}^{-1}} d \tau\right),
\end{gathered}
$$

with the function $A_{0}(\mathbf{x})$ satisfying (28). The solution (29) has an interesting feature in the case when $k_{1} \neq 0, A_{0}(\mathbf{x}) \in L_{2}\left(\mathbb{R}^{n}\right)$ and $a_{0}<0$. At a finite time, $T=-a_{0}^{-1}$, the mass concentrates at a point which escapes to infinity.

If we suppose that $A_{0}(\mathbf{x}) \mathbf{x} \in L_{2}\left(\mathbb{R}^{n}\right)$, we can express the solution through the functionals $M(t), Q_{\Lambda}(t)$ and $\tilde{\mathbf{P}}$. Indeed, for the velocity field (24) we have

$$
\begin{gathered}
M^{\prime}(t)=2 a(t) M(t)+b(t) Q_{\Lambda}(t), \\
\tilde{P}_{\Lambda}=a(t) Q_{\Lambda}(t)+b(t) N|\boldsymbol{\Lambda}|^{2} .
\end{gathered}
$$

Further, from (3), (30), (31) we obtain

$$
a^{\prime}(t)=a(t) \frac{2 \tilde{P}_{\Lambda} Q_{\Lambda}(t)-M^{\prime}(t) N|\boldsymbol{\Lambda}|^{2}}{M(t) N|\boldsymbol{\Lambda}|^{2}-Q_{\Lambda}^{2}(t)}-\frac{\tilde{P}_{\Lambda}^{2}-8 H N|\boldsymbol{\Lambda}|^{2}}{M(t) N|\boldsymbol{\Lambda}|^{2}-Q_{\Lambda}^{2}(t)} .
$$

Then we use (4) and (10) to get

$$
a^{\prime}(t)=-a(t) \frac{F^{\prime}(t)}{F(t)}+\frac{C}{F(t)},
$$

where $F(t)=A t^{2}+B t+C, A=8 H N|\boldsymbol{\Lambda}|^{2}-\tilde{P}_{\Lambda}^{2}, B=M^{\prime}(0) H N|\boldsymbol{\Lambda}|^{2}-2 \tilde{P}_{\Lambda} Q_{\Lambda}(0)$, $C=M(0) N|\boldsymbol{\Lambda}|^{2}-Q_{\Lambda}^{2}(0)$. Note that by virtue of the Hölder inequality $C \geq 0$. 
Further, we find from (32) and (31)

$$
\begin{aligned}
a(t) & =\frac{C t}{F(t)}+a_{0}, \\
b(t) & =\frac{\tilde{P}_{\Lambda}(A-C) t^{2}+\left(\tilde{P}_{\Lambda}\left(B-a_{0}\right)-C Q_{\Lambda}(0)\right) t+\left(\tilde{P}_{\Lambda} C-a_{0} Q_{\Lambda}(0)\right)}{F(t) N|\boldsymbol{\Lambda}|^{2}} .
\end{aligned}
$$

\section{ACKNOWLEDGMENTS}

This work was partially supported by the Russian Foundation for Basic Researches Award No. 03-02-16263.

\section{REFERENCES}

1. J.Ginibre, G.Velo, The global Cauchy problem for the nonlinear Schrödinger equation revisited. Ann.Inst.H.Poincaré. Anal.Non Lineare 2(1985), no.4, 309-327. MR.0801582 (87b:35150)

2. J.Ginibre, G.Velo, On a class of nonlinear Schrödinger equations. J.Funct.Anal. 32(1979), no.1, 1-32. MR0533219 (82c:35058)

3. T.Kato, On nonlinear Schrödinger equations. Ann.Inst.H.Poincaré. Phys.Theor. 46(1987), no.1, 113-129. MR0877998 (88f:35133)

4. T.Cazenave, F.Weissler. Some remarks on the nonlinear Schrödinger equation in the critical case. Nonlinear semigroups, partial differential equations and attractors (Washington, DC, 1987), 18-29, Lecture Notes in Math., Springer, Berlin-New York, 1989. MR1021011 (91a:35149)

5. B.Birnir, C.Kenig, G.Ponce, N.Svanstedt, L.Vega, On the ill-posedness of the IVP for the generalized Korteweg-de Vries and nonlinear Schrödinger equations. J.London Math. Soc.(2) 53(1996), no.3, 551-559. MR,1396718 (97d:35233)

6. M.I.Weinstein, Nonlinear Schrödinger equations and sharp interpolation estimates. Comm.Math.Phys. 87(1982/83), no.4, 567-576. MR0691044(84d:35140)

7. R.T.Glassey, On the blowing up of solutions to the Cauchy problem for nonlinear Schrödinger equations. J.Math.Phys. 18(1977), no.9, 1794-1797. MR0460850|(57:842)

8. F.Merle, Blow up phenomena for critical nonlinear Schrödinger and Zakharov equations. Documenta Mathematica, Extra volume ICM 1998, III (1998), 57-66. MR.1648140 (99h:35200)

9. V.I.Talanov, Self-focusing of wave beams in nonlinear media. JETP Lett. 2(1965), 138.

10. T.Ogawa, Y.Tsutsumi, Blow-up of $H^{1}$ solutions for the nonlinear Schrödinger equation. J. Differential Equations 92(1991), no.2, 317-330. MR.1120908 (92k:35262)

11. H.Nawa, Asymptotic and limiting profiles of blowup solutions of the nonlinear Schrödinger equation with critical power. Comm.Pure Appl. Math., 1999, v.52, no.2, 191-270. MR1653454 (99m:35235)

12. E.Madelung, Quantentheorie in hydrodynamischer form, Z.Phys. 40(1926), 322.

13. D.Serre, Solutions classiques globales des équations d'Euler pour un fluide parfait compressible. Annales de l'Institut Fourier 47(1997), 139-153. MR1437182 (98a:35108)

14. O.S.Rozanova, Classes of smooth solutions to multidimensional balance laws of gas dynamic type on Riemannian manifolds. Trends in mathematical physics research, 155-204, Nova Sci. Publ., Hauppauge, NY, 2004. MR2078813

15. O.S.Rozanova, Solutions with linear profile of velocity to the Euler equations in several dimensions. Hyperbolic problems: Theory, numerics, applications, 861-870, Springer, Berlin, 2003. MR2053233

16. O.S.Rozanova, Application of integral functionals to the study of the properties of solutions to the Euler equations on riemannian manifolds, J.Math.Sci. 117(5)(2003), 4551-4584. MR2027444 (2004k:76018)

17. Y.Tsutsumi, Rate of $L^{2}$ - concentration of blow up solutions for the Nonlinear Schrödinger equation with critical power. Nonlinear Analysis, Methods \& Application 15(1990), no.8, 719724. MR1074950 (91j:35043)

18. H.Berestycki, P.-L.Lions, Nonlinear scalar field equation. I,II. Arch.Rational Mech.Anal. 82(1983), no.4, 313-345 and 347-375. MR0695535(84h:35054a) MR0695536 (84h:35054b) 
19. M.I. Weinstein. On the structure and formation of singularities in solutions to nonlinear dispersive evolution equations, Communications in Partial Differential Equations 11(1986), no.5, 545-565. MR0829596 (87i:35026)

20. F.Merle, Determination of blow-up solutions with minimal mass for nonlinear Schrödinger equation with critical power. Duke Math. J. 69(1993), no.2, 427-454. MR1203233 (94b:35262)

21. F.Merle, On uniqueness and continuation properties after blow-up time of self-similar solutions of nonlinear Schrödinger equation with critical exponent and critical mass. Comm. Pure Appl. Math. 45(1992), no.2, 203-254. MR1139066 (93e:35104)

22. W.-M.Ni, R.D.Nussbaum, Uniqueness and nonuniqueness for positive radial solutions of $\Delta u+$ $f(u, r)=0$. Comm. on Pure Appl. Math., 1985, v.38, 67-108. MR0768105|(86d:35055)

Department of Differential Equations, Mathematics and Mechanics Faculty, Moscow State University, GSP-2 Vorobiovy Gory, Moscow 119992, Russia

E-mail address: rozanova@mech.math.msu.su 